\title{
Transbronchial Lung Cryobiopsy in the Diagnosis of Interstitial Lung Diseases. Looking at the Holy Grail or Another Misleading Diagnostic Approach?
}

\author{
Ioannis Sotiriou, Alaxandra Konstantinidou \\ University Hospital of Larissa, Larissa, Greece \\ Email: giasotiriou@yahoo.gr
}

How to cite this paper: Sotiriou, I. and Konstantinidou, A. (2018) Transbronchial Lung Cryobiopsy in the Diagnosis of Interstitial Lung Diseases. Looking at the Holy Grail or Another Misleading Diagnostic Approach? Open Journal of Respiratory Diseases, 8, 69-74.

https://doi.org/10.4236/ojrd.2018.84008

Received: September 27, 2018

Accepted: November 9, 2018

Published: November 12, 2018

Copyright $\odot 2018$ by authors and Scientific Research Publishing Inc. This work is licensed under the Creative Commons Attribution International License (CC BY 4.0).

http://creativecommons.org/licenses/by/4.0/

\begin{abstract}
Interstitial lung diseases (ILD's) are a group of heterogenous chronic, ferociously progressive lung diseases. The aetiology of the aforementioned diseases is not always recognisable. The diagnosis of these dismal diseases is a vivid challenge for the physicians. Through the intervening years different diagnostic algorithms have been implemented towards more accurate outcome. Different types of ILD's demand diverse diagnostic approaches. In the latest years a novel diagnostic mini invasive approach seems to gain continuously terrain towards the diagnosis of ILD's. Transbronchial cryobiopsy may be the Holy Grail in the diagnosis of these diseases or a misleading diagnostic tool in this challenging field.
\end{abstract}

\section{Keywords}

Transbronchial Lung Biopsy, Transbronchial Lung Cryobiopsy, Surgical Lung Biopsy, Interstitial Lung Diseases

\section{Introduction}

ILD's are many in number, accounting more than 200 different types. However the most frequently seen are less than ten, Idiopathic pulmonary fibrosis, Cryptogenic organizing pneumonia (COP), Desquamative interstitial pneumonitis, Nonspecific interstitial pneumonitis, Hypersensitivity pneumonitis, Acute interstitial pneumonitis, Interstitial pneumonia, Sarcoidosis, Asbestosis, Respiratory Bronchiolitis Interstitial Lung Disease (RB-ILD). The flagship and perhaps, the leading disease is the idiopathic pulmonary fibrosis (IPF). The latter carries 
the most dismal prognosis. The incidence and prevalence of IPF fluctuates between 1.25 to 23.4 cases per 100,000 population [1]. In Europe, the annual incidence ranged between 0.22 and 7.4 per 100,000 population. We, thankfully, have an updated clinical practice guidelines coming from a fruitful collaboration of ATS/ERS/JRS/ALAT, for the diagnosis of IPF [2]. The latest, of course, is of great help trying to adopt a contemporary and sufficient way to diagnose and manage the aforementioned disease. However, this will be a theoretical tool in the hands of the physicians being involved in the management of ILD's. To further establish the diagnosis a tissue confirmation would be appropriate in certain circumstances.

The practical guidance coming from the published guidelines refined the classification of the pathognomonic pattern of usual interstitial pneumonia (UIP). That includes the typical appearance of the UIP on High Resolution Computed Tomography (HRCT) analysis. Further stratification of the endoparenchymal architectural distortions, leads to the rest of subtypes: propable UIP, Indeterminate and alternative diagnosis. The different types have different diagnostic approach. The gold standard in the diagnosis of IPF still remains the newly identified appearances on HRCT, of UIP pattern. However, with regards to the above mentioned refined subtypes, because of lack of evidence, no recommendation was made for or against performing transbronchial lung biopsy or lung cryobiopsy. Having in mind this valid, efficacious and well-designed, volume of recommendations, we could be more confident in the ILD's management. But are we?

\section{Tissue Confirmation Challenge}

The major and possibly the most challenging part in the management is when it comes to the point where obtaining a tissue for confirmation of the diagnosis is unavoidable. As far as the IPF concerns, the guidance is firmed. What about the rest of the diffused parenchymal lung diseases (DPLD's)?

Is surgical approach still remains the Gold standard towards diagnosis of not determined DPLD's via the cross section images? Or the cryobiopsies are equally efficacious and accurate?

There is a robust interest in the latest years about the wider implementation of the aforementioned technique in the diagnostic algorithm of the ILD's. A novel technique always sparks the immediate hope of resolving usually challenging problems. However this requires a focused, pragmatic well controlled and standardised approach in order to avoid unpredictable outcomes.

There is a very interesting input, in fact an experts statement, in the literature coming from the Cryobiopsy Working Group on Safety and Utility of the Procedure. They have utilized their concerns on further standardization of the procedure, taking into consideration different aspects. The careful and profound enrolment of the right cohort of patients, the pathological status, contraindications and the safety profile of the procedure per se, are few of the major concerns 
that demands further attention [3].

Analysis of the results performed from studies from diverse centres, utilizing the procedure in the diagnosis of ILD's, have shown a reasonable number of positive outcomes with regards to the confirmation of the diagnosis. The safety profile and the diagnostic yield were found to be similar to Surgical Lung Biopsies (SLB). However the final establishment of the diagnosis required a profound multidisciplinary approach by confident experts in the field [4].

Regardless of the growing interest and the number of centres getting involved and adopting the technique as part of the diagnostic algorithm in ILD's, the lack of robust common path in utilizing the technique is still in its infancy [5]. The willingness to accept and adopt a novel technique was and always will be a milestone for better understanding the disease and optimizing the therapy. However this is not enough in real world clinical settings. Common notion, in performing the technique, certain lines of mutual guidance, creating a wide registry with the outcome of the procedure and multicentre involvement in actual comparison with SLB should all be implemented for better outcomes.

\section{Pitfalls in Accuracy of TBLB vs TBLC}

Another fragile and sensitive issue in the path of this technique to be further established and gain its place as a valuable diagnostic tool, is the numbers and frequency of complications (Table 1). As invasive technique is not free of significant complications. There is evidence coming from published studies were the results from cryobiopsies were profoundly good, raging more than $80 \%$ in definite diagnosis. Moreover, the complications observed were no significant and further less was the need of escalating the diagnostic algorithm with SLB [5] [6]. In contrast there is evidence of bleeding being the second commonest adverse event following pneumothorax in certain series [7].

The observed range of similarities or diversities in the nature of the complications or the number of these, can be explained by the lack of a definite, solid way of the technique been utilised. Secondly, the absence of narrow and mutually agreed sensitive threshold, with regards to the pool of patients eligible to be enrolled in the procedure (one size doesn't fit all). Last but not least, the absence of a more distinct guidance on contraindications.

Accuracy of the diagnosis fundamentally relies upon the number of the obtained specimens and their dimensions as well. As an invasive diagnostic tool

Table 1. TBLC versus conventional TBLB.

\begin{tabular}{cccccc}
\hline Author & Year & $\begin{array}{c}\text { Disease } \\
\text { involved }\end{array}$ & Type of study & $\begin{array}{c}\text { Specimen size } \\
\text { TBLC/TBLB }\end{array}$ & $\begin{array}{c}\text { Diagnostic yield\% } \\
\text { TBLC/TBLB }\end{array}$ \\
\hline Badiak & 2009 & ILD's & Retrospective & $15.11 \mathrm{~mm}^{2} / 5.82 \mathrm{~mm}^{2}$ & $95.12 / 58.53$ \\
Griff & 2011 & ILD & $\begin{array}{c}\text { Retrospective } \\
\text { Clinical trial }\end{array}$ & $17.1 \mathrm{~mm}^{2} / 3.8 \mathrm{~mm}^{2}$ & $92.7 / 78$ \\
Pajares & 2014 & ILD & $\begin{array}{c}\text { Randomized trial } \\
14.7 \mathrm{~mm}^{2} / 3.3 \mathrm{~mm}^{2}\end{array}$ & $74.4 / 34.1$ \\
\hline
\end{tabular}


Transbronchial Lung Cryobiopsy (TBLC) showed some signs of priority versus the transbronchial lung biopsies (TBLB). Namely, the mean number of the tissue obtain from the latter was 4 per patient and from the former 2 per patient. On the other hand the dimensions of the specimens retrieved from the TBLC were larger than these from TBLB $(0.6 \mathrm{~cm}-2.6 \mathrm{~cm}$ vs $0.1-0.8 \mathrm{~cm}$ respectively) [8]. This of course is a credit for TBLC. However, not few of the enrolled patients underwent SLB for final establishment of the diagnosis. Complications, once further, were pneumothorax and haemorrhage. Retaining the undebatable superiority of the TBLC as less invasive procedure than SLB, could be said that the diagnostic dilemma as to which approach to be chosen for the diagnosis is somehow resolved. But is it? [9] [10] [11].

\section{Dynamics of TBLC vs SLB}

Unquestionably, SLB have been a crucial tool in the diagnosis of ILD's. The physicians involved in the management of the aforementioned disorders, relied on the latter surgical approach. That has helped to deliver optimal management and precise therapeutic options. However, given the fact of continuously growing demand of less invasive techniques to be implemented in the diagnostics, as to minimize the burden of complication and the use of extra resources, TBLC might be considered a promising method. Further reports are suggesting the latter to be safe and adequate method for the diagnosis of ILD's (Table 2). This has been accompanied by as high as $74 \%$ success in establishing the final diagnosis. Additionally the complications observed were manageable and less life-threatening [12] [13].

\section{Discussion}

The evidences up to date with regards to the usefulness of TBLC as a diagnostic tool in ILD's are pointing towards a feasible and in general, safe method. Should this method be decided as a valuable alternative to surgical approach in the diagnosis of ILD's, then physicians should be instructed more precisely on its use. Wider and more uncontrolled apply of the technique might result in inflated burden of complications and worse outcomes.

Table 2. Safety profile in SLB versus TBCB.

\begin{tabular}{ccc}
\hline Complications & SLB $(\mathrm{n}=150)$ & TBLC $(\mathrm{n}=297)$ \\
\hline Severe Bleeding & 0 & 0 \\
Persistent fever & 7 & 0 \\
Prolonged airleak & 5 & 1 \\
Acute exacerbation & 5 & 1 \\
Pneumonia/Empyema & 3 & 0 \\
Transient respiratory failure & 0 & 2 \\
Pneumothorax (in Total) & NA & 60 \\
Pneumothorax requiring Drainage & NA & 46 \\
\hline
\end{tabular}


The future research should focus in expanding a safer protocol with universal applying methodology. That could be achieved by enabling this unique and novel diagnostic approach in centres of excellence and following appropriate randomized and prospective studies in order to ensure accurate and optimal reproducible results.

The history has shown that we have a propensity in implementing novel strategies being in its infancy, applying them in clinical settings though. There have been advances in the results and outcomes in the intervening years with regards to the safety and efficacy of TBLC. However, we have still way to go prior to suggest this could be a definite alternative to SLB. Well-designed studies are required before deciding the most appropriate diagnostic approach in ILD's. Profound adherence to the stratification of the right cohort of patients is another crucial factor. Implementation of alternative diagnostic approaches without reproducible or with less accurate results, may be a misleading tool and not the Holy Grail in the challenging field of the interstitial lung diseases.

\section{Conflicts of Interest}

The authors declare no conflicts of interest regarding the publication of this paper.

\section{References}

[1] Nalysnyk, L., et al. (2012) Incidence and Prevalence of Idiopathic Pulmonary Fibrosis: Review of the Literature. European Respiratory Review, 21, 355-361. https://doi.org/10.1183/09059180.00002512

[2] Raghu, G., et al. (2018) Diagnosis of Idiopathic Pulmonary Fibrosis. An Official ATS/ERS/JRS/ALAT Clinical Practice Guideline. American Journal of Respiratory and Critical Care Medicine, 198, e44-e68. https://doi.org/10.1164/rccm.201807-1255ST

[3] Hetzel, J., et al. (2018) Transbronchial Cryobiopsies for the Diagnosis of Diffuse Parenchymal Lung Diseases: Expert Statement from the Cryobiopsy Working Group on Safety and Utility and a Call for Standardization of the Procedure. Respiration, 9, 188-200. https://doi.org/10.1159/000484055

[4] Lentz, R.J., Taylor, T.M., Kropski, J.A., Sandler, K.L., Johnson, J.E., Blackwell, T.S., Maldonado, F. and Rickman, O.B. (2018) Utility of Flexible Bronchoscopic Cryobiopsy for Diagnosis of Diffuse Parenchymal Lung Diseases. The Journal of Bronchology and Interventional Pulmonology, 25, 88-96.

[5] Lentz, R.J., et al. (2017) Transbronchial Cryobiopsy for Diffuse Parenchymal Lung Disease: A State-of-the-Art Review of Procedural Techniques, Current Evidence, and Future Challenges. Journal of Thoracic Disease, 9, 2186-2203. https://doi.org/10.21037/jtd.2017.06.96

[6] Kropski, J.A., et al. (2013) Bronchoscopic Cryobiopsy for the Diagnosis of Diffuse Parenchymal Lung Disease. PLoS One, 8, e78674.

https://doi.org/10.1371/journal.pone.0078674

[7] Bango-Álvarez, A., et al. (2017) Transbronchial Cryobiopsy in Interstitial Lung Disease: Experience in 106 Cases-How to Do It. ERJ Open Research, 3, 00148-2016. https://doi.org/10.1183/23120541.00148-2016 
[8] Sriprasart, T., Aragaki, A., Baughman, R., Wikenheiser-Brokamp, K., Khanna, G., Tanase, D., Kirschner, M. and Benzaquen, S. (2017) A Single US Center Experience of Transbronchial Lung Cryobiopsy for Diagnosing Interstitial Lung Disease with a 2-Scope Technique. Journal of Bronchology and Interventional Pulmonology, 24, 131-135. https://doi.org/10.1097/LBR.0000000000000366

[9] Babiak, A., Hetzel, J., Krishna, G., Fritz, P., Moeller, P., Balli, T. and Hetzel, M. (2009) Transbronchial Cryobiopsy: A New Tool for Lung Biopsies. Respiration, 78, 203-208. https://doi.org/10.1159/000203987

[10] Griff, S., et al. (2011) Morphometrical Analysis of Transbronchial Cryobiopsies. Diagnostic Pathology, 6, 53. https://doi.org/10.1186/1746-1596-6-53

[11] Pajares, V., et al. (2014) Diagnostic Yield of Transbronchial Cryobiopsy in Interstitial Lung Disease: A Randomized Trial. Respirology, 19, 900-906.

ttps://doi.org/10.1111/resp.12322

[12] Ramaswamy, A., Homer, R., Killam, J., Pisani, M.A., Murphy, T.E., Araujo, K. and Puchalski, J. (2016) Comparison of Transbronchial and Cryobiopsies in Evaluation of Diffuse Parenchymal Lung Disease. Journal of Bronchology \& Interventional Pulmonology, 23, 14-21. https://doi.org/10.1097/LBR.0000000000000246

[13] Ravaglia, C., Bonifazi, M., Wells, A.U., Tomassetti, S., Gurioli, C., Piciucchi, S., Dubini, A., Tantalocco, P., Sanna, S., Negri, E., Tramacere, I., Ventura, V.A., Cavazza, A., Rossi, A., Chilosi, M., La Vecchia, C., Gasparini, S. and Poletti, V. (2016) Safety and Diagnostic Yield of Transbronchial Lung Cryobiopsy in Diffuse Parenchymal Lung Diseases: A Comparative Study versus Video-Assisted Thoracoscopic Lung Biopsy and a Systematic Review of the Literature. Respiration, 91, 215-227. https://doi.org/10.1159/000444089

\section{Abbreviation List}

ILD: Interstitial Lung Diseases;

TBCB: Transbronchial Cryobiopsy;

TBLC: Transbronchial Lung Biopsy;

SLB: Surgical Lung Biopsy. 